\title{
CRENÇAS DE ENFERMEIROS DO CONTEXTO HOSPITALAR SOBRE A INCLUSÃO DA FAMÍLIA NO CUIDADO DE ENFERMAGEM
}

\author{
NURSES' BELIEFS OF THE HOSPITAL CONTEXT ABOUT THE INCLUSION OF THE FAMILY IN \\ NURSING CARE
}

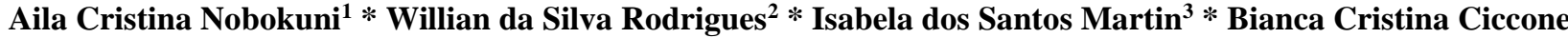

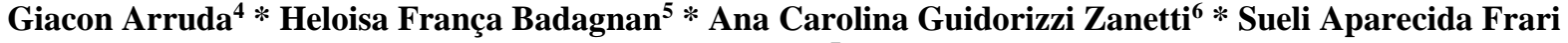 \\ Galera $^{7}$
}

\begin{abstract}
RESUMO
Objetivo: O objetivo deste estudo foi descrever as crenças de enfermeiros hospitalares sobre a inclusão da família no planejamento do cuidado de enfermagem. Métodos: Estudo qualitativo realizado com 10 enfermeiros hospitalares por meio de entrevista áudio gravada, submetidas a análise de conteúdo temática. A pesquisa foi autorizada por comitê de ética em pesquisa com seres humanos. Resultados: Duas categorias emergiram da análise dos dados: A presença da família no contexto hospitalar e ambulatorial promove a melhora do paciente, fortalece o vínculo familiar e é uma oportunidade para o enfermeiro fazer ensinamentos e, algumas famílias têm dificuldades para se envolver no cuidado do paciente. Discussão: os enfermeiros reconheceram a importância da presença do familiar como um apoio ao paciente facilitando sua recuperação física e mental e veem na internação a possibilidade de oferecer orientações para o cuidado após a alta. Porém também foi apontado que algumas famílias te dificuldades de se envolver ao cuidado por vários motivos além disso algumas famílias são vistas como questionadoras pela equipe. Considerações finais: $\mathrm{O}$ estudo atendeu aos objetivos propostos, mais estudos são recomendados para conhecer as atitudes e crenças dos enfermeiros em outros cenários de atenção à saúde. Esse estudo possibilitou enriquecer a discussão a cerca do trabalho da enfermagem com as famílias dos pacientes.
\end{abstract}

Palavras-chave: Percepção; Cuidados de Enfermagem; Atitude; Família; Enfermagem Familiar.

\begin{abstract}
Objective: The objective of this study was to describe the beliefs of hospital nurses about the inclusion of the family in nursing care planning. Methods: Qualitative study that was carried out with 10 hospital nurses through recorded audio interviews, submitted to thematic content analysis. The research was authorized by the Human Research Ethics Committee. Results: Two categories emerged from the data analysis: The presence of the family in the hospital and outpatient context promotes patient improvement, strengthens the family bond and is an opportunity for nurses to teach and, some families have difficulties to get involved in the care of the patient. Discussion: nurses recognized the importance of the presence of the family member as a support for the patient, facilitating their physical and mental recovery and see in the hospitalization the possibility of offering guidance for care after discharge. However, it was also pointed out that some families find it difficult to get involved in care for various reasons, other than that some families are seen as questioners by the team. Final considerations: The study met the proposed objectives, more studies are recommended to learn about nurses attitudes and beliefs in other health care settings. This study made it possible to enrich the discussion about nursing work with the patients families.
\end{abstract}

Keywords: Perception; Nursing Care; Attitude; Family; Family Nursing.

\footnotetext{
${ }^{1}$ Enfermeira. Mestranda pelo Departamento de Enfermagem Psiquiátrica e Ciências Humanas da Escola de Enfermagem de Ribeirão Preto da Universidade de São Paulo. ORCID: 0000-0001-7952-316X. E-mail: aila.nobokuni@usp.br

${ }^{2}$ Psicológo, mestre pelo Departamento de Enfermagem Psiquiátrica e Ciências Humanas da Escola de Enfermagem de Ribeirão Preto da Universidade de São Paulo. ORCID: 0000-0001-5353-0011. E-mail: willpsico7@ outlook.com

${ }^{3}$ Enfermeira. Enfermeira no Departamento de Enfermagem Psiquiátrica e Ciências Humanas da Escola de Enfermagem de Ribeirão Preto da Universidade de São Paulo. ORCID: 0000-0001-8274-4641. E-mail: ismartin@ eerp.usp.br

${ }^{4}$ Enfermeira. Professora Doutora pela Universidade Federal de Mato Grosso do Sul. ORCID: 0000-0002-8433-6008. E-mail: biagiacon@gmail.com

${ }^{5}$ Enfermeira. Doutora pelo Departamento de Enfermagem Psiquiátrica e Ciências Humanas da Escola de Enfermagem de Ribeirão Preto da Universidade de São Paulo. ORCID: 0000-0001-9087-7671. E-mail: heloisabadagnan @ yahoo.com.

${ }^{6}$ Enfermeira. Professora Doutora no Departamento de Enfermagem Psiquiátrica e Ciências Humanas da Escola de Enfermagem de Ribeirão Preto da Universidade de São Paulo. ORCID: 0000-0003-0011-4510. E-mail: carolzan @eerp.usp.br

${ }^{7}$ Enfermeira. Professora Doutora no Departamento de Enfermagem Psiquiátrica e Ciências Humanas da Escola de Enfermagem de Ribeirão Preto da Universidade de São Paulo. ORCID: 0000-0001-7974-9214. E-mail: sugalera@usp.br
} 


\section{INTRODUÇÃO}

A família é a célula vital para a saúde de seus membros, é onde aprendemos a cuidar da saúde e da doença e enfrentar os desafios da $\operatorname{vida}^{(1)}$. A teoria dos sistemas familiares, reconhece que uma situação de doença, ou de risco para a saúde afeta todos os membros da família. Reciprocamente, as relações familiares podem contribuir para as formas de gerenciar a saúde da família. O reconhecimento desta interação, reciprocidade e relacionamentos convida os pesquisadores a buscarem mudanças na maneira de pensar e praticar o cuidado, partindo de um modelo de cuidar apenas do paciente individualmente para considerar a família como paciente ${ }^{(2,3)}$.

Entre os profissionais de saúde a enfermagem é a que mais tem contato com pacientes e suas famílias ${ }^{(4)}$. Por esta razão tem mais oportunidade para envolve-la na assistência em saúde nos diferentes contextos de cuidado. Para a enfermagem envolver a família dos pacientes ao planejar e prestar cuidados é um compromisso inerente à profissão ${ }^{(4)}$. Apesar desta posição privilegiada a enfermagem ainda enfrenta muitos desafios para tal prática. Esses desafios podem estar relacionados à cultura organizacional da instituição e da equipe de saúde que podem favorecer ou não a presença da família. A formação pessoal do enfermeiro e sua capacidade de compreender a importância do envolvimento familiar ao cuidado também são fatores que influenciam a inclusão da família no cuidado ${ }^{(5-6)}$.

A atitude do enfermeiro em relação à família é outro aspecto que pode influenciar a prática da enfermagem familiar ${ }^{(7)}$. Atitude envolve os componentes afetivo, cognitivo e comportamental, sendo considerada uma resposta a um estímulo ${ }^{(8)}$. Na enfermagem, ela pode ser definida como o conjunto de práticas, conhecimentos, habilidades, estratégias e satisfação que o enfermeiro tem para incluir a família no seu trabalho por meio do planejamento do cuidado, promovendo a participação da família e reconhecendo as dificuldades e os facilitadores na relação terapêutica, respeitando suas singularidades $(8,9)$.

As crenças do enfermeiro sobre o papel da família na saúde de seus membros é elemento fundamental para a construção de uma atitude que facilite a interação enfermeiro-familia. As crenças dos enfermeiros sobre a eficiência do cuidado familiar podem facilitar e favorecer o sucesso da implementação da enfermagem familiar nos serviços de atendimento à saúde ${ }^{(10)}$.

Quando os enfermeiros mantêm uma postura descrente e não apresentam atitudes e práticas voltadas para a inclusão da família no seu plano de cuidados, geralmente não procuram realizar um trabalho centrado no cuidado familiar. Assim, podem não perceber os benefícios de fazê-lo ${ }^{(6,10)}$. 
Deste modo questiona-se: Como os enfermeiros de um contexto hospitalar percebem a família de seus pacientes e quais as crenças envolvidas no trabalho com as famílias? Assim, o objetivo deste estudo foi descrever quais as crenças de enfermeiros hospitalares sobre a inclusão da família no planejamento do cuidado de enfermagem.

\section{MÉTODOS}

Estudo qualitativo, fundamentado na compreensão de que a atitude do enfermeiro frente à família envolve componentes afetivos, cognitivos e comportamentais que se refletem em sua prática profissional ${ }^{(1,7)}$. As crenças sobre o papel da família no cuidado constituem o elemento cognitivo da atitude ${ }^{(8,10)}$. Para a construção deste trabalho utilizamos o roteiro Consolidated criteria for reporting qualitative research $\mathrm{COREQ}^{(11)}$.

Os participantes do estudo são enfermeiros que trabalham em um hospital escola de nível terciário em um município no interior do Estado de São Paulo. Foram escolhidos enfermeiros que atuam em unidade onde há a presença de familiares e excluídos aqueles que estavam em período probatório ou atuavam em setores que não tinham contato com famílias. O período estabelecido para a coleta de dados foi de abril a maio de 2018. Doze enfermeiros foram convidados para participar do estudo e, dois não aceitaram alegando que não tinham tempo disponível para responder as perguntas. Assim, participaram do estudo 10 enfermeiros que atuavam em unidades de geriatria, clínica médica, psiquiatria, transplante de medula óssea, cardiologia, neonatologia, neurologia e ginecologia.

Os dados foram coletados por meio de entrevistas individuais, audiogravadas que tiveram duração de 15 a 40 minutos. Todas as entrevistas foram realizadas por uma única pesquisadora, graduanda em enfermagem e treinada em coleta de dados, por meio da participação em um grupo de pesquisa e por acompanhamento de entrevistas e atendimentos realizados por membros do grupo. Esse treinamento foi supervisionado por uma enfermeira doutora, também pesquisadora e autora deste trabalho. A entrevistadora não conhecia os participantes.

$\mathrm{O}$ instrumento de coleta de dados foi constituído de duas partes. Um questionário de caracterização sociodemográfica dos enfermeiros: sexo; estado civil e se possuía algum tipo de pós-graduação. Para a condução da entrevista foram usadas três perguntas abertas: "Que problemas ou inconvenientes há em sua prática de enfermagem ao envolver a família na avaliação e planejamento dos cuidados?"; "Quais são os benefícios, se existir algum, de incluir a família na sua prática de enfermagem?"; e "O que você fez na semana passada para envolver as famílias na sua prática de enfermagem atual? Por favor 
comente.” Estas perguntas compõem parte do instrumento Family Nursing Practice Scale (FNPS) desenvolvida em Hong $\mathrm{Kong}^{(8)}$ e adaptada culturalmente e validada em seus aspectos psicométricos para o português do Brasil $^{(12)}$.

Este estudo foi cumpriu todos os preceitos éticos e foi autorizado pelo Comitê de Ética em Pesquisa em Seres Humanos da Escola de Enfermagem de Ribeirão Preto da Universidade de São Paulo em 23 de março de 2018, sob número de protocolo CAAE:77821317.1.0000.5395. Após a aprovação foi iniciada a coleta de dados. A pesquisadora entrou em contato pessoalmente com os enfermeiros durante sua jornada de trabalho para apresentar a proposta da pesquisa e solicitar a participação. Após acordado o melhor momento para a participação, foi apresentado o Termo de Consentimento Livre e Esclarecido (TCLE) que foi assinado em duas vias, uma do participante e outra da pesquisadora. Em seguida, o participante preencheu $\mathrm{o}$ questionário sociodemográfico e respondeu a entrevista, realizada em ambiente privativo na unidade de atuação do participante. Permaneceram no local apenas o participante e a pesquisadora. Cada participante respondeu apenas uma entrevista.

As entrevistas foram submetidas a análise de conteúdo temática conforme descrita por Bardin ${ }^{(13)}$. As entrevistas foram transcritas na íntegra. Todas as falas que pudessem identificar os participantes ou outras pessoas do ambiente de trabalho foram ocultadas de modo a preservar a identidade de todos. Depois de várias leituras, iniciou-se a leitura linha a linha de cada entrevista com a finalidade de identificar palavras-chave ou frases que representassem aspectos da atitude do entrevistado em relação à inclusão da família no planejamento do cuidado de enfermagem. Ao lado de cada trecho identificado, era redigida uma observação sobre o entendimento do pesquisador sobre aquele aspecto.

O passo seguinte foi reunir esses trechos em grupos semelhantes e novamente era redigido uma observação expressando o entendimento do pesquisador sobre aquele grupo de frases ou palavras-chave. O próximo estágio da análise envolveu reunir as categorias semelhantes em temas mais amplos. Esse processo foi realizado por três pesquisadores de maneira independente. Uma reunião do grupo permitiu encontrar as semelhanças e diferenças no entendimento de cada um e a busca de consenso sobre as categorias e temas desenvolvidos ${ }^{(13)}$.

\section{RESULTADOS}

Foram entrevistados dez enfermeiros oito do sexo feminino. Quatro eram casados, dois eram divorciados, um estava em uma união estável e três eram solteiros. Cinco enfermeiros relataram que possuíam pós- 
graduação ou alguma especialização, sendo as áreas: Enfermagem oncológica, estoma terapia, transplante e doação de órgãos, pedagogia e saúde mental, e um enfermeiro concluiu o mestrado em transplante de órgãos.

A análise das entrevistas permitiu compreender que os enfermeiros entendem que a inclusão da família no cuidado de enfermagem se dá por meio da presença e participação do familiar no cuidado prestado ao adoecido no contexto hospitalar e no domicílio. A presença do familiar durante a internação ou no atendimento ambulatorial favorece a recuperação do adoecido e permite que o enfermeiro ensine o cuidado que deve ser continuado no domicílio. Potencializando assim o papel da família na recuperação do adoecido. Por outro lado, os enfermeiros destacaram que algumas famílias têm dificuldade de se envolver com o cuidado do adoecido, sendo, portanto, uma barreira para sua recuperação.

Ao articularmos as categorias identificadas neste estudo percebemos que elas descrevem as crenças dos enfermeiros sobre o papel da família nos cuidados com o familiar adoecido. Esta compreensão é descrita em duas crenças apresentadas por meio de afirmações.

A presença da família no contexto hospitalar e ambulatorial promove a melhora do paciente, fortalece o vínculo familiar e é uma oportunidade para o enfermeiro fazer ensinamentos
Todos os enfermeiros que participaram deste estudo acreditam que a presença e a participação do familiar no cuidado durante a internação promovem uma melhora mais rápida e efetiva do paciente.

Quando a família se envolve, o resultado é muito melhor em relação ao tratamento. Mesmo que as vezes o paciente é um paciente difícil, um paciente que não adere ao tratamento, mas se tem envolvimento da família muita das vezes a gente consegue um resultado muito melhor, diminuição das taxas de internação, menos visitas ao ambulatório por descompensação, então assim é muito gratificante pra mim quanto profissional, para meu trabalho ser eficiente, a eficácia minha é quando os pacientes vêm nos ambulatórios $e$ não interna. (E8)

Em psiquiatria se não tem suporte, não tem como, você vai perder tempo, e política de saúde em psiquiatria ter um suporte familiar, a gente sabe que nem todo mundo tem, mas é fundamental que tenha, para ter sucesso, os protocolos em psiquiatria deveriam ser fundamentais, caso ele não tenha, você tem que fazer o melhor possivel, ver se ele tem amigo, ou vizinho, alguém que se responsabilize. (E3)

A presença da família no serviço de saúde também é vista como uma oportunidade de ensinar a família sobre os cuidados que o adoecido necessitará em casa. Para os enfermeiros, quanto mais a família for 
preparada para o cuidado, menor serão as reinternações e melhor o prognostico e evolução da doença

Quando a família se envolve, o resultado é muito melhor em relação ao tratamento. Mesmo que as vezes o paciente é um paciente dificil, um paciente que não adere ao tratamento, mas se tem envolvimento da família muita das vezes a gente consegue um resultado muito melhor, diminuição das taxas de internação, menos visitas ao ambulatório por descompensação, então assim é muito gratificante pra mim quanto profissional, para meu trabalho ser eficiente, a eficácia minha é quando os pacientes vem nos ambulatórios $e$ não interna. (E8)

Eu acho que quando a gente consegue explicar algumas coisas pra família, nem sempre, mas a grande maioria das vezes, a gente consegue dar, nem sei se um tratamento melhor é a palavra, mas um retorno mais demorado pra vir pro serviço. (E4)

O adoecido pode se sentir muito inseguro no contexto da internação, pode pensar que está abandonado. A presença do familiar acompanhando a internação pode fortalecer o vínculo entre o paciente e sua família, favorecer a auto estima do adoecido que se sente amado e protegido pelo familiar.

A recuperação é mais rápida (quando o familiar participa do cuidado), o paciente fica com autoestima elevada $e$ a recuperação é um sucesso e muito mais rápida, o processo do tratamento e a eficácia do mesmo se torna mais efetiva, o paciente que se torna mais efetivo, mais amado, que tem uma visão de um tratamento da doença dele, diferente daquele que não tem, quando a pessoa se sente importante ela se sente valorizada, agora se ela sente que não tem importância, ela não liga pra nada, a partir do momento que ela se sente valorizada, o rendimento dela é melhor, a resposta ao tratamento é melhor. (E3)

Observou-se que precisa haver uma disposição do enfermeiro para ensinar a família a cuidar em casa. Indicando que quando o enfermeiro acredita nessa oportunidade de ensinar a família e o faz ela retribui aderindo aos cuidados. A adesão da familia aos ensinamentos do enfermeiro reforçam nele a crença sobre a importância da familia no cuidado do adoecido.

Então você se dispõe a ensinar o familiar a cuidar em casa da úlcera do paciente, até você receber o paciente de volta sem a lesão. Minha maior retribuição é ver o paciente curado, é ver que o paciente ter melhora, melhora na fala, melhora na mobilidade, em tudo, então a olhos vistos é você ver o paciente melhorar, então quando a família que está muito empenhada, que é uma família que interage, você consegue prestar os cuidados de um planejamento que a enfermagem $e$ que a equipe medica se propõe, esse paciente tem ganhos né, então isso é muito gratificante. (E10) 
Nesse sentido, uma das práticas dos enfermeiros com a família é fornecer os ensinamentos que os familiares precisam para prestar o cuidado adequado em casa. Para isso, foi referida a necessidade de $\mathbf{o}$ enfermeiro observar melhor os membros da família. Como exemplo o enfermeiro busca identificar qual o melhor familiar para receber orientações e, com isso, adquirir mais as informações e experiência sobre $\mathrm{O}$ adoecimento do paciente e os cuidados necessários.

Às vezes, o paciente tem três filho. Um é nervoso, o outro chora e o outro consegue receber e passar informação de forma simples. Eu convoco a família e durante a conversa eu observo muito aquele membro que menos se movimenta, que fica quietinho observando todos os recados, que presta atenção em você pra passar pra família. Eu sempre tento eu tento inserir essa pessoa de qualquer forma independente do diagnóstico, tanto se for bom, quanto se for ruim. (E2)

O envolvimento da família no processo de orientação do enfermeiro sobre o cuidado ao paciente é visto, pelos enfermeiros, como primordial, principalmente para a alta hospitalar.

A alta...Ela é programada, ninguém acorda e recebe a notícia que vai de alta naquele dia, ela é programada uns dias antes, as vezes até uma semana antes, porque tem a questão dos medicamentos que a gente precisa ter a certeza que o paciente vai ter acesso, tem as orientações que precisamos ter certeza que o paciente $e$ acompanhante entenderam, o paciente aqui nunca é responsável sozinho pelo seu tratamento, precisa sempre de uma pessoa junto. Então as orientações sempre são dadas para o paciente e acompanhante. (E7)

Bom, muitas vezes o problema pra gente é a falta de estrutura familiar, muitas vezes na nossa orientação para insuficiência cardíaca é importante o envolvimento familiar, porque como é muito complexo o que ele tem que fazer em casa, por exemplo, peso diário em jejum após a primeira diurese, restrição hídrica que é muito complicado, restrição sódica, isso tudo o paciente não consegue fazer sozinho se não tiver o apoio da família, então muitas vezes nós esbarramos nesse problema, a falta de estrutura familiar ou a falta até de família, tem pacientes que são institucionalizados, ou a família é muito ausente, isso é um problema muito grave pra gente, porque esse paciente eventualmente volta pras internações.(E8)

\section{Algumas famílias têm dificuldades para se envolver no cuidado do paciente.}

Embora reconheçam que a família é importante na recuperação do adoecido, alguns enfermeiros relataram que muitas vezes as famílias não se envolvem com o cuidado sugerido na orientação de 
enfermagem. Isso acontece, segundo eles, por diferentes motivos como baixa escolaridade e capacidade de compreensão, terem que trabalhar e não conseguirem estar mais próximos durante a internação ou no retorno do ambulatório, sobrecarga do cuidado, entre outros.

Essas barreiras identificadas pelos enfermeiros exigem que eles realizem adequações na sua prática. Estas adequações envolvem ajustar o vocabulário ao nível educacional do familiar flexibilidade para conseguir incluir a família.

Problemas? Problemas que as vezes eles não estão presentes né, nas atividades do dia a dia, as vezes a falta de clareza deles, nos demanda mais clareza na hora de passar informação e .... acho que mais basicamente isso. Em especial, eu não tive não, nenhum grande problema, mas como lidamos com pessoas menos esclarecidas né, uma renda mais baixa, então automaticamente o nível de entendimento é menor, mas especificamente eu tento falar mais claramente. (E6)

Os enfermeiros citam também que outras famílias comparecem esporadicamente aos retornos ou ao acompanhamento do paciente, por não compreender a importância dela nesse processo. Estes contextos foram descritos como como situações de tensão entre o enfermeiro e a família, o que interfere na prática com famílias.
Muitas vezes não fica (o familiar não fica na internação ) porque tem outros problemas, trabalha $e$ tal não pode ficar, mas quando pode, mesmo assim tem famílias que não ficam, E1)

As vezes eles (os familiares) vêm uma única vez, não sabe como funciona e ai fica nervoso, quer chutar, quer bater porque não sabe como funciona, e a mesma coisa de quem tem câncer, então a maior dificuldade é a falta de entendimento deles, e quando eles não acompanham, vem em consultas esporádica. (E9)

As vezes tem três filhos e um é nervoso, o outro chora e o outro consegue receber a informação do medico e passar de forma simples, porque as vezes o filho a ansiedade dele, ele recebe a noticia e passa ela para os outros de um jeito completamente distorcido pra família (E2)

Na enfermaria a família questiona o porquê de tudo as vezes não comparecia durante a alta $e$ internação, então eles sempre colocavam empecilho para não fazer o que a gente pedia após a alta, aqui não é diferente. (E5)

Algumas famílias foram descritas como questionadoras. Questionam sempre para que serve o medicamento que está sendo administrado, perguntam sobre os horários e sobre as atividades da equipe de enfermagem. Estas famílias são consideradas participativas, porém os questionamentos podem ser vistos como uma atitude inibidora da interação com 
a enfermagem, dificultando que sejam feitas as orientações necessárias.

Então eu já escutei coisas assim: A se eu ficar lá de acompanhante eu tenho que ajudar, eu tenho que trocar, eu tenho que fazer, ai eles não fazem, quando na verdade eu fico me questionando se a pessoa não consegue realizar a interpretação de que talvez aquela situação não melhore $e$ que todos os cuidados que estão sendo prestados aqui (no hospital), terão que ser realizados em casa, e ai como que vai ser esses cuidados em casa? Sendo que eles não enxergam o fato de nos ajudar como uma oportunidade de aprendizado que vai ser seguido em casa entendeu?. (E1)

Eu me sinto muito cobrada, seja pela equipe, ou pelos familiares, eles cobram coisas como curativos, exames que pra eles se falam as 7:00 da manhã, as 7:01 já querem estar fazendo o exame, não entendem que tem uma ordem, uma fila, sem perceber as vezes, claro que as vezes não consegue mesmo perceber que existe uma dinâmica de trabalho e que existem outros pacientes $e$ que as vezes não tem profissional pra tudo isso, ai a gente até entende no início que o familiar não tem essa ideia mais às vezes esse comportamento da família acaba ficando quando a internação é longa e eles já deveriam ter percebido que muitas vezes não é questão de falta de vontade que as coisas não são feitas de imediato e que de uma forma ou de outra ele precisa ficar um pouco independente desse cuidado de enfermagem, porque na casa dele será que ele vai ter essa ajuda?. (E1)

\section{DISCUSSÃO}

Esta pesquisa identificou que os participantes acreditam que a família é importante no cuidado seu ente adoecido. Quando a família participa do cuidado ela favorece os vínculos familiares, melhora a autoestima do adoecido, favorece sua adesão ao tratamento. A presença da família foi considerada uma oportunidade para que o enfermeiro faça ensinamentos sobre os cuidados com o adoecido. Neste contexto a presença da família e sua interação com a enfermagem promove a recuperação física e mental mais rápida e satisfatória do adoecido, colaborando também no sentimento de valorização do enfermeiro. Além de reforçar a crença sobre a importância da familia no cuidado do adoecido. Por outro lado, reconhecem que algumas famílias têm dificuldades de se envolver no cuidado do adoecido.

A literatura aponta para este aspecto da interação entre a enfermagem e a família como elemento primordial para a recuperação de pessoas adoecidas. Os profissionais de enfermagem são os que permanecem mais tempo ao lado do paciente e seu familiar durante a internação, oferecendo, em suas rotinas diárias, orientações sobre o tratamento, procedimentos e cuidados necessários ${ }^{(3-4)}$. 
Destaca-se, porém, que a inclusão do familiar no cuidado de enfermagem ainda é vista somente como uma oportunidade de fazer orientações e ensinamentos sobre como cuidar. Assim a equipe mantém a crença de que a inclusão da família está relacionada ao cuidado cotidiano, sem a preocupação sobre como e em que condições estes cuidados serão executados no domicílio ${ }^{(14-16)}$.

Essas crenças dos enfermeiros podem estar relacionadas com a pouca proximidade destes profissionais com a abordagem dos sistemas familiares, que reconhece que quando um dos membros da família é afetado por uma doença todo o sistema se desestabiliza ${ }^{(1,7)}$. A falta de conhecimento sobre esta abordagem pode ser reflexo de um ensino tradicional da enfermagem que é voltado para o cuidado individual em detrimento de uma abordagem familiar ${ }^{(10,15)}$.

Um estudo misto realizado na Holanda com 18 enfermeiros hospitalares ofereceu um treinamento de seis dias sobre os sistemas familiares. Os autores puderam ver que os profissionais perceberam a importância de ter uma visão sistêmica do grupo familiar e que ao adotar esta abordagem podem melhorar o relacionamento entre equipe, paciente e seu parente e a rotina profissional. Pois torna possível planejar e otimizar o tempo de trabalho com um diálogo e intervenções mais efetivas $^{(15)}$.

Duhamel et al destacam que é importante investir no ensino de enfermeiros sobre a abordagem dos sistemas familiares. Os conceitos e ferramentas desta abordagem devem ser introduzidos respeitando o conhecimento prévio dos profissionais, que devem ser incentivados a aplicar o novo conhecimento junto com aquilo que já conhecem. Assim, a medida que os conhecimentos e ações específicos vão sendo agregados ao dia a dia, e os profissionais percebem resultados positivos dessas ações podem mudar suas crenças e influenciar na inclusão e trabalho com as famílias e pacientes. Resultados positivos junto com auto reforço são um importante fator motivacional para continuar aprendendo $\mathrm{e}$ praticando o cuidado integral para ambos ${ }^{(1)}$.

Um dos resultados obtidos neste estudo é de que os enfermeiros veem no familiar uma possibilidade de realizar orientações e conhecimentos sobre os cuidados que irão ser continuados em casa após a alta. Essa visão unilateral como a internação como oportunidade de fazer ensinamento desconsidera todo o contexto familiar onde o cuidado ensinado deverá ocorrer. Isto é, este parente é compreendido como um agente de continuidade do cuidado do hospital para o domicílio, porém essa continuidade é uma preocupação do profissional que não leva em consideração as condições familiares para a efetivação do cuidado ${ }^{(15-16)}$.

Outro aspecto a ser considerado é que familiares que possuem um parente 
hospitalizado encontram-se em uma situação de fragilidade biopsicossocial. Uma vez que, a necessidade de internação é marcada por eventos que geram desconfortos e dúvidas sobre a melhora e recuperação do adoecido. As inseguranças e medos podem favorecer para que o parente não se sinta confortável e não permaneça presente durante a internação (17). Este aspecto não foi considerado pelos participantes desta pesquisa, que perceberam o familiar sempre como alguém para dar continuidade aos cuidados no domicilio.

Pode-se pensar que a dificuldade dos enfermeiros de conversar com os familiares sobre assuntos que vão além da orientação também seja reflexo do medo de invadir o espaço privado da família ou de se aproximar de aspectos emocionais para os quais o enfermeiro não se sente preparado. Segundo a literatura o receio de aproximação emocional com a família do doente, pode ser vista como prejudicial para o desempenho do enfermeiro $(18,19)$.

Porém, quando a família, por algum motivo, é questionadora, ou seja, é vista pelo enfermeiro como alguém que pressiona de alguma forma a equipe, ela perde a oportunidade de receber os ensinamentos e orientações que irão auxiliar no cuidado em casa. Esse comportamento é referido como causador de conflito entre a equipe, que podem ser intensificados devido ao grande período de internação, a dedicação total do parente ao adoecido onde é necessário deixar temporariamente a rotina já estabelecida, além da preocupação com a condição de saúde e acerca dos procedimentos enfrentados pelo adoecido $^{(19-20)}$.

\section{CONSIDERAÇÕES FINAIS}

O objetivo desse estudo foi descrever crenças de enfermeiros hospitalares sobre a inclusão da família no planejamento do cuidado de enfermagem. O método adotado permitiu identificar essas crenças e relacionála com as atitudes e práticas dos participantes da pesquisa. Os participantes percebem que a família é importante para dar apoio emocional ao adoecido e poder receber as orientações sobre o cuidado que será continuado em casa. A presença da família promove a recuperação do adoecido. Porém também apontaram algumas dificuldades no trabalho com as famílias, como as ausências e os questionamentos excessivos.

O número reduzido de participantes pode ser uma limitação do estudo que foi causado pelo curto espaço de tempo para a coleta de dados. Porém os relatos obtidos nas entrevistas e a análise cuidadosa por pesquisadores distintos de forma independente possibilitaram dar maior credibilidade ao conteúdo analisado. Mais estudos precisam ser realizados para conhecer não só as crenças e atitudes de enfermeiros em outros cenários de atuação, mas também os desafios dessa prática, barreiras e 
facilidades, podendo assim enriquecer a discussão sobre os benefícios do cuidado pautado nos sistemas familiares.

\section{REFERÊNCIAS}

1. Duhamel F, Dupuis F, Turcotte A, Martinez AM, Goudreau J. Integrating the illness beliefs model in clinical practice: A family systems nursing knowledge utilization model. J Fam Nurs [Internet]. 2015[acesso em 18 de maio de 2021]; 21(2):322-48. Disponível em: https://doi.org/10.1177/1074840715579404

2. Grácio J, Gonçalves PM, Leff J. Key elements of a family intervention for schizophrenia: a qualitative analysis of an RCT. Fam Process [Internet]. 2018 [acesso em 17 de maio 2021]; 57(1):100-12. Disponível em: https://pubmed.ncbi.nlm.nih.gov/27896805/

3. Nóbrega MPSS, Fernandes CSNN, Angelo M, Chaves SCS. Importance of families in nursing care for people with mental disorders: attitudes of Portuguese and Brazilian nurses. Rev Esc Enferm USP [Internet]. 2020 [acesso em 17 de maio 2021]; 54:e03594. Disponível em:

https://www.scielo.br/scielo.php?script=sci_ar ttext\&pid=S0080-

\section{0\&tlng=en}

4. Wright L, Bell JM. Enfermeiras Famílias: Guia para avaliação e intervenção na Família. 5. Ed. São Paulo:Roca; 2015
5. Farias DHR, Gomes GC, Almeida MFF, LechLunardi V, Xavier DM, Queiroz MVO.Barreiras presentes no processo de construção do cuidado familiar cultural à criança no hospital: abordagem transcultural*. Aquichan[Internet]. 2019 [acesso em 17 de maio 2021]; 19(1):2. Disponível em: https://dialnet.unirioja.es/servlet/articulo?codi go $=7075162$

6. Cruz AC, Pedreira MLG. Patient-and Family-Centered Care and Patient Safety: Reflections upon Emerging Proximity. Rev Bras Enferm [Internet]. 2020 [acesso em 17 de maio 2021]; 73(6):e20190672. Disponível em:

https://www.scielo.br/scielo.php?pid=S0034$71672020000600403 \&$ script=sci_arttext

7. Fernandes CS, Gomes JAP, Martins MM, Gomes BP, Gonçalves LHT. The Importance of Families in Nursing Care: Nurses' Attitudes in the Hospital Environment. Rev Enf Ref [Internet]. 2015 [acesso em 17 de maio 2021]; serIV(7):21-30. Disponível em: http://www.scielo.mec.pt/scielo.php?script=sc i_arttext\&pid=S0874-02832015000700003

8. Simpson P, Tarrant M. Development of the Family Nursing Practice Scale. J Fam Nurs [Internet]. 2006 [acesso em 25 de janeiro de 2021]; 12(4):413-25. Disponível em: https://pubmed.ncbi.nlm.nih.gov/17099118/

9. Hagedoorn EI, Paans W, Jaarsma T, Keers JC, Van Der Schans CP, Luttik ML, et al. Translation and Psychometric Evaluation of the Dutch Families Importance in Nursing 
Care: Nurses' Attitudes Scale Based on the Generalized Partial Credit Model. J Fam Nurs[Internet]. 2018[ acesso em 17 de maio 2021]; 24(4):538-62. Disponível em: $10.1177 / 1074840718810551$.

10. Rice H, Howard R, Huntley J. Professional caregivers' knowledge, beliefs and attitudes about awareness in advanced dementia: a systematic review of qualitative studies. Int Psychogeriatr [Internet]. 2019 [acesso em 18 de maio 2021]; 31(11):1599609. Disponível

em: https://pubmed.ncbi.nlm.nih.gov/30789113/

11. Equator. Enhancing the QUAlity and Transparency Of health Research [Internet].2020[cited 2021 maio 17]; Disponível em: https://www.equatornetwork.org/reporting-guidelines/coreq/

12. Rodrigues WDS, Badagnan HF, Nobokuni AC, Fendrich L, Zanetti ACG, Giacon BCC, Galera SAF. Family Nursing Practice Scale: Portuguese Language Translation, Cross-Cultural Adaptation, and Validation. J Fam Nurs[Internet].2021[acesso em 17 de maio 2021].Disponível em : https://doi.org/10.1177/10748407211002152

13. Bardin L. Análise do Conteúdo. 229p. São Paulo: Edições 70; 2011.

14. Cruz AC, Angelo M. Good relationships with families in the neonatal and pediatric context: defi nition from the nurses' perspective. Rev Soc Bras Enferm Pediatras [Internet]. 2018 [acesso em 17 de maio de 2021]; 18(2):69-77. Disponível em: https://journal.sobep.org.br/en/article/goo d-relationships-with-families-in-the-neonataland-pediatric-context-definition-from-thenurses-perspective/

15. Broekema S, Luttik MLA, Steggerda GE, Paans W, Roodbol PF. Measuring change in nurses' perceptions about family nursing competency following a 6-day educational intervention. J Fam Nurs[Internet]. 2018[acesso em 18 de maio de 2021]; 24(4):508-37. Disponível em: https://doi.org/10.1177/1074840718812145

16. De Macedo IF, De Souza TV, Dos Santos Oliveira IC, Cibreiros SA, De Morais RDCM, Vieira RFC. As concepções da equipe de enfermagem frente à família da criança hospitalizada. Rev Bras Enferm[Internet]. 2017 [acesso em 18 de maio 2021]; 70(5):952-60. Disponível em: https://www.redalyc.org/articulo.oa?id=26705 2669003

17. Uema RTB, Bega AG, Rodrigues BC, Lopes APAT, Hiragashi IH, Decesaro MN. Family-centered care in the perspective of nurses from an adult hospitalization unit. Ciênc cuid saúde [Internet]. 2017 [acesso em 17 de maio de 2021]; 16(4):1-7. Disponível

em: http://www.periodicos.uem.br/ojs/index.p hp/CiencCuidSaude/article/view/37466

18. Farias DHR, Gomes GC, Almeida MFF, Lunardi VL, Xavier DM, Queiroz MVO. Barriers Present in the Process of Construction of the Cultural Family Care to 
the Child in the Hospital: Transcultural Approach. Aquichan [Internet]. 2019 [acesso em 17 de maio de 2021]; 19(1):e1912. Disponível

em: https://pesquisa.bvsalud.org/portal/resource/pt /biblio-1011135

19. Queiroz TA, Ribeiro ACM, Guedes MVC, Coutinho DTR, Galiza FT, Freitas MC. Palliative care to the elderly in intensive care: the perspective of the nursing team. Texto \& contexto enferm [Internet]. 2018 [acesso em 17 de maio de 2021]; 27(1):e1420016. Disponível em: https://www.scielo.br/scielo.php?script=sci_a bstract\&pid=S0104$07072018000100310 \& \operatorname{lng}=$ en $\& n r m=$ iso 20.Buoro RS, Nogueira MP. Quality of life and challenges of family members of children with meningomyelocele. Acta ortop bras
[Internet]. 2020 [acesso em 17 de maio 2021]; 28(6):291-95. Disponível em: https://www.scielo.br/scielo.php?script=sci_a bstract\&pid=S1413-

$78522020000600291 \& \operatorname{lng}=\mathrm{en} \& \mathrm{nrm}=\mathrm{iso}$

Submissão: 2021-02-08

Aprovado: 2021-05-19

${ }^{1}$ Escola de Enfermagem de Ribeirão Preto da Universidade de São Paulo.

Avenida dos Bandeirantes, 3900, Campus Universitário - Bairro Monte Alegre

Ribeirão Preto - SP - Brasil. CEP: 14040-902.

2 Universidade Federal de Mato Grosso do Sul-Avenida Marcio Lima Nantes, s/n. Vila da Barra. Coxim - MS- Brasil. CEP: 79400000 